\title{
A new exact quantum mechanical propagator
}

\author{
F W Wiegel and $P$ W van Andel \\ Center for Theoretical Physics, Twente University of Technology, PO Box 217, Enschede \\ 7500 AE, The Netherlands
}

Received 8 May 1986

\begin{abstract}
We derive a closed-form expression for the time-dependent propagator of a quantum mechanical particle which is subject to an external force which is the sum of (i) a reflecting half-plane barrier with a straight edge, and (ii) a harmonic force pointing towards a point of the edge. This new addition to the short list of exactly known quantum mechanical Green functions is a simple combination of exponential functions and Fresnel integrals, the arguments of which are combinations of trigonometric functions.
\end{abstract}

\section{General considerations}

For only very few quantum mechanical problems is the time-dependent propagator known explicitly. The subject of this paper is the addition of one more example to this short list.

Consider a particle of mass $m$, with cartesian coordinates $x, y, z$. Let this quantum mechanical particle be subject to two forces:

(i) a reflecting half-plane barrier at $-\infty<x<0, y=0,-\infty<z<+\infty$, and

(ii) a harmonic force with a potential

$$
V(x, y, z)=\frac{1}{2} m \omega^{2}\left(x^{2}+y^{2}\right)+\frac{1}{2} m \omega_{0}^{2} z^{2} .
$$

Note that the force constants for the $x$ and $y$ components of the motion must be equal to each other. The propagator $G\left(x, y, z, t \mid x_{0}, y_{0}, z_{0}\right)$ for this problem can be written as the product of a propagator $(g)$ for the projection of the motion onto the $x, y$ plane and the propagator for the projection of the motion onto the $z$ axis. As the reflecting half-plane barrier does not influence the $z$ component of the motion, the propagator for the $z$ component is the one for the harmonic oscillator. This gives

$$
\begin{aligned}
& G\left(x, y, z, t \mid x_{0}, y_{0}, z_{0}\right) \\
&= g\left(x, y, t \mid x_{0}, y_{0}\right)\left(\frac{2 \pi i \hbar}{m \omega_{0}} \sin \left(\omega_{0} t\right)\right)^{-1 / 2} \\
& \times \exp \left(\frac{\mathrm{i} m \omega_{0}}{2 \hbar} \frac{\left(z^{2}+z_{0}^{2}\right) \cos \left(\omega_{0} t\right)-2 z z_{0}}{\sin \left(\omega_{0} t\right)}\right)
\end{aligned}
$$

so the non-trivial part of the problem is the calculation of $g$.

We shall calculate $g$ following the method of Wiegel and Boersma (1983) and Wiegel (1986), which derives the quantum mechanical propagator from a specific 
(physically unrelated!) polymer entanglement problem. This paper is organised as follows. In $\S 2$ the relevant polymer entanglement problem and its previously derived solution are briefly discussed. This solution is used in $\S 3$ to find the solution of various problems concerning the paths of a Brownian particle in the presence of an absorbing medium but in the absence of a screen. In that section we shall also separate the Brownian motion paths into 'even' and 'odd' paths and calculate their weights separately. These results make it possible in $\$ 4$ to calculate the propagator of a certain diffusion problem; the quantum mechanical propagator $g$ follows by transforming this propagator to imaginary time. Some concluding remarks are collected in $\S 5$.

\section{A relevant polymer entanglement problem}

Instead of the cartesian coordinates in the $x, y$ plane it will turn out to be more convenient to introduce polar coordinates $r, \theta$ in that plane. Let $0<r<\infty$ and $-\pi<\theta<$ $+\pi$. The initial position $\left(x_{0}, y_{0}\right)$ has polar coordinates $\left(r_{0}, \theta_{0}\right)$, the final position $(x, y)$ has polar coordinates $(r, \theta)$. The half-plane barrier intersects this plane in a 'branchline' $T$ with polar coordinates $0<r<\infty, \theta= \pm \pi$; the geometry is shown in figure 1 .

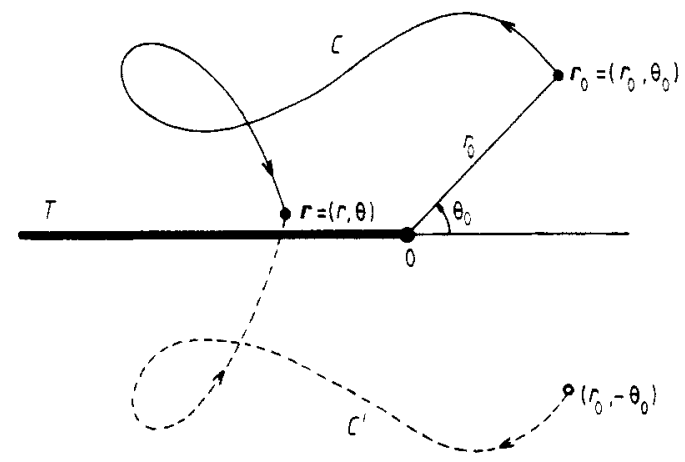

Figure 1. Geometry of the two-dimensional entanglement problem. $C$ and $C^{\prime}$ are polymer chains ( $\$ 2$ ) or Brownian motion paths ( $\$ 3$ and 4 ). The branchline $T$ is the intersection of the half-plane barrier with the $(r, \theta)$ plane. The harmonic force is centred at the origin of the $(r, \theta)$ plane. In the limit $\theta \uparrow \pi, C$ and $C^{\prime}$ are each other's mirror image with respect to the straight line through $T$ : if $C$ is even (odd), then $C^{\prime}$ is odd (even).

Now consider the following polymer entanglement problem which is physically completely unrelated to the quantum mechanical problem under consideration. A polymer chain consists of $N$ freely hinged rods, each of length $l$. The chain is constrained to this plane and has fixed initial and final positions at $\left(r_{0}, \theta_{0}\right)$ and $(r, \theta)$. The repeating units of the chain are not subject to the reflecting half-plane barrier, but they are subject to an external force with a potential $V(r)$ which is a function of the radial distance $r$ of the form

$$
V(r)=\frac{1}{2} k_{\mathrm{B}} T m^{\prime} \omega^{2} r^{2}
$$

where $k_{\mathrm{B}}$ denotes Boltzmann's constant and $T$ the absolute temperature. The relation between $m$ and $m^{\prime}$ will be clarified shortly. 
As the polymer chain is not subject to the reflecting half-plane barrier its configurations can be entangled with the $z$ axis (i.e. the origin of coordinates in the $x, y$ plane) a number of times. To precisely specify the entanglement we introduce the branchline $T$ (which is now not hard!). For a given polymer configuration $C$ the entanglement index $n$ is defined as

$$
n(C)=n_{+}(C)-n_{-}(C)
$$

where $n_{+}(C)=0,1,2, \ldots$ denotes the number of times that $C$ crosses $T$ in the direction of increasing $\theta$ and $n_{-}(C)=0,1,2, \ldots$ denotes the number of passages in the opposite direction.

Now, let $Q_{n}(r, \theta, N)$ denote the configuration sum of the polymer, restricted to those configurations which start in $\left(r_{0}, \theta_{0}\right)$, end in $(r, \theta)$ and have an entanglement index $n$. It was shown by Wiegel (1977) that each of the functions $Q_{n}$ with $n=$ $0, \pm 1, \pm 2, \ldots$ is a solution of the partial differential equation:

$$
\left(\partial / \partial N-\frac{1}{4} l^{2} \Delta+\frac{1}{2} m^{\prime} \omega^{2} r^{2}\right) Q_{n}(r, \theta, N)=0
$$

where $N$ is treated as a continuous variable and where $\Delta$ denotes the two-dimensional Laplace operator with respect to the coordinates of the endpoint. It was also shown that the various $Q_{n}$ are related to each other on $T$ by certain unusual boundary conditions, and that

$$
\begin{aligned}
\lim _{N \rightarrow 0} Q_{n} & =\delta\left(x-x_{0}\right) \delta\left(y-y_{0}\right) & & \text { if } n=0 \\
& =0 & & \text { if } n \neq 0 .
\end{aligned}
$$

The explicit calculation of $Q_{n}$ was performed by Inomata and Singh (1978) and Tanikella and Inomata (1982) using path integral techniques and by van Andel (1986) using the last two equations. They find

$$
\begin{aligned}
Q_{n}(r, \theta, N)= & \frac{\omega}{\pi l}\left(\frac{m^{\prime}}{2}\right)^{1 / 2} \operatorname{cosech}\left[N l \omega\left(\frac{m^{\prime}}{2}\right)^{1 / 2}\right] \\
& \times \exp \left\{-\frac{\omega}{l}\left(\frac{m^{\prime}}{2}\right)^{1 / 2}\left(r_{0}^{2}+r^{2}\right) \operatorname{coth}\left[N l \omega\left(\frac{m^{\prime}}{2}\right)^{1 / 2}\right]\right\} \\
& \times \int_{-x}^{+\infty} I_{|k|}\left[2 \frac{\omega}{l}\left(\frac{m^{\prime}}{2}\right)^{1 / 2} r_{0} r \operatorname{cosech}\left[N l \omega\left(\frac{m^{\prime}}{2}\right)^{1 / 2}\right]\right. \\
& \times \exp \left[i k\left(\theta-\theta_{0}+2 \pi n\right)\right] \mathrm{d} k
\end{aligned}
$$

where $I_{\nu}$ denotes the modified Bessel function of order $\nu$.

An independent check of this result is to consider the limit $\omega \rightarrow 0$. One finds

$\lim _{\omega \rightarrow 0} Q_{n}(r, \theta, N)=\left(\pi N l^{2}\right)^{-1} \exp \left(-\frac{r_{0}^{2}+r^{2}}{N l^{2}}\right) \int_{-\infty}^{+\infty} I_{\mid k}\left(\frac{2 r_{0} r}{N l^{2}}\right) \exp \left[\mathrm{i} k\left(\theta-\theta_{0}+2 n \pi\right)\right] \mathrm{d} k$

which is indeed the correct expression for the simple entanglement problem, of the review by Wiegel (1983). Another independent check of (2.5) will be presented in the next section. 


\section{Entanglement properties of Brownian motion paths in an absorbing medium}

We now introduce the auxiliary variable $\tau_{0}$ with the dimension of time and take the limits

$$
\begin{aligned}
& N \rightarrow \infty \\
& l \rightarrow 0 \\
& \tau_{0} \rightarrow 0 \\
& m^{\prime} \rightarrow 0
\end{aligned}
$$

such that the three combinations

$$
\begin{aligned}
& N \tau_{0}=t \\
& l^{2} / 4 \tau_{0}=D \\
& m^{\prime} / \tau_{0}=m
\end{aligned}
$$

remain constant. In this limit the polymer configurations turn into the paths of a particle which performs Brownian motion in the $x, y$ plane with a diffusion coefficient $D$. The particle moves in an absorbing medium; the probability of annihilation equals $\frac{1}{2} m \omega^{2} r^{2}$ per unit of time. Indeed, the configuration sums $Q_{n}(r, \theta, N)$ change into probabilities of survival $Q_{n}(r, \theta, t)$ with continuous time $t$. By dividing (2.3) by $\tau_{0}$ it is seen that $Q_{n}(r, \theta, t)$ is a solution of

$$
\left(\partial / \partial t-D \Delta+\frac{1}{2} m \omega^{2} r^{2}\right) Q_{n}(r, \theta, t)=0
$$

subject to the appropriate boundary conditions and to the initial condition

$$
\begin{aligned}
\lim _{t \rightarrow 0} Q_{n} & =\delta\left(x-x_{0}\right) \delta\left(y-y_{0}\right) & & \text { if } n=0 \\
& =0 & & \text { if } n \neq 0 .
\end{aligned}
$$

Taking the limit (3.1) and (3.2) in (2.5) one finds for the probability that a Brownian motion path still survives at time $t$ and has an entanglement index $n$ with the origin:

$$
\begin{aligned}
Q_{n}(r, \theta, t)=\frac{\omega}{\pi} & \left(\frac{m}{8 D}\right)^{1 / 2} \operatorname{cosech}\left[(2 m D)^{1 / 2} \omega t\right] \\
& \times \exp \left[-\omega\left(\frac{m}{8 D}\right)^{1 / 2}\left(r_{0}^{2}+r^{2}\right) \operatorname{coth}\left[(2 m D)^{1 / 2} \omega t\right]\right] \\
& \times \int_{-\infty}^{+\infty} I_{k}\left[\omega\left(\frac{m}{2 D}\right)^{1 / 2} r_{0} r \operatorname{cosech}\left[(2 m D)^{1 / 2} \omega t\right]\right] \\
& \times \exp \left[i k\left(\theta-\theta_{0}-2 \pi n\right)\right] \mathrm{d} k .
\end{aligned}
$$

One can use this result to derive various corollaries. It will turn out to be useful to explicitly evaluate the sum of $Q_{n}$ over even values of $n$, as well as the sum over odd values of $n$. A Brownian motion path which crosses the branchline $T$ an even number of times will be called an even path, and the sum over all even paths will be denoted by $E\left(r, \theta, t \mid r_{0}, \theta_{0}\right)$. Similarly, the sum over all odd paths will be denoted by $O\left(r, \theta, t \mid r_{0}, \theta_{0}\right)$.

In order to calculate the total probability of the even paths we note that

$$
E\left(r, \theta, t \mid r_{0}, \theta_{0}\right)=\sum_{n^{\prime}=-\infty}^{+\infty} Q_{2 n^{\prime}}(r, \theta, t)
$$


Substituting (3.5), and using the summation formula

$$
\sum_{n^{\prime}=-\infty}^{+\infty} \exp \left(4 \pi n^{\prime} k \mathrm{i}\right)=\frac{1}{2} \sum_{l=-\infty}^{+\infty} \delta\left(k-\frac{l}{2}\right)
$$

one finds

$$
\sum_{n^{\prime}=-\infty}^{+\infty} \int_{-\infty}^{+\infty} I_{|k|}(z) \exp \left[\mathrm{i} k\left(\theta-\theta_{0}+4 \pi n^{\prime}\right)\right] \mathrm{d} k=\frac{1}{2} I_{0}(z)+\sum_{i=1}^{\infty} I_{\frac{1}{2}}(z) \cos \frac{1}{2} l\left(\theta-\theta_{0}\right)
$$

where

$$
z=\omega\left(\frac{m}{2 D}\right)^{1 / 2} r_{0} r \operatorname{cosech}\left[(2 m D)^{1 / 2} \omega t\right] .
$$

The sum on the right-hand side of (3.8) can be performed by writing

$$
\begin{aligned}
\sum_{l=1}^{\infty} I_{1} l & (z) \cos \frac{1}{2} l\left(\theta-\theta_{0}\right) \\
= & \sum_{l^{\prime}=1}^{\infty} I_{l^{\prime}}(z) \cos l^{\prime}\left(\theta-\theta_{0}\right)+\sum_{l^{\prime}=0}^{\infty} I_{r^{\prime}+\frac{1}{2}}(z) \cos \left(l^{\prime}+\frac{1}{2}\right)\left(\theta-\theta_{0}\right) .
\end{aligned}
$$

The first term is given by equation (9.6.34) of Abramowitz and Stegun (1970):

$$
\sum_{l^{\prime}=1}^{\infty} I_{l^{\prime}}(z) \cos l^{\prime}\left(\theta-\theta_{0}\right)=\frac{1}{2} \exp \left[z \cos \left(\theta-\theta_{0}\right)\right]-\frac{1}{2} I_{0}(z) \text {. }
$$

The second term was calculated explicitly in equation (13) of Wiegel and Boersma (1983):

$$
\sum_{l^{\prime}=0}^{\infty} I_{l^{\prime}+\frac{1}{2}}(z) \cos \left(l^{\prime}+\frac{1}{2}\right)\left(\theta-\theta_{0}\right)=\frac{1}{2} \exp \left[z \cos \left(\theta-\theta_{0}\right)\right] \operatorname{erf}\left[(2 z)^{1 / 2} \cos \frac{1}{2}\left(\theta-\theta_{0}\right)\right]
$$

where erf stands for the error function

$$
\operatorname{erf}(\xi)=\frac{2}{\sqrt{ } \pi} \int_{0}^{\xi} \mathrm{e}^{-\eta^{2}} \mathrm{~d} \eta
$$

Collecting all these results the total probability of the even paths is found to be given by the expression

$$
E\left(r, \theta_{1}, t \mid r_{0}, \theta_{0}\right)=\frac{1}{2} z^{\prime \prime} \exp \left[z \cos \left(\theta-\theta_{0}\right)-z^{\prime}\right]\left\{1+\operatorname{erf}\left[(2 z)^{1 / 2} \cos \frac{1}{2}\left(\theta-\theta_{0}\right)\right]\right\}
$$

where

$$
\begin{aligned}
& z^{\prime}=\omega\left(\frac{m}{8 D}\right)^{1 / 2}\left(r_{0}^{2}+r^{2}\right) \operatorname{coth}\left[(2 m D)^{1 / 2} \omega t\right] \\
& z^{\prime \prime}=\frac{\omega}{\pi}\left(\frac{m}{8 D}\right)^{1 / 2} \operatorname{cosech}\left[(2 m D)^{1 / 2} \omega t\right] .
\end{aligned}
$$

The total probability of the odd paths can be calculated in a similar way and is found to be given by

$$
\begin{aligned}
O\left(r, \theta, t \mid r_{0}, \theta_{0}\right) & \\
& =\sum_{n^{\prime}=-\infty}^{+\infty} Q_{2 n^{\prime}+1}(r, \theta, t) \\
& =\frac{1}{2} z^{\prime \prime} \exp \left[z \cos \left(\theta-\theta_{0}\right)-z^{\prime}\right]\left\{1-\operatorname{erf}\left[(2 z)^{1 / 2} \cos \frac{1}{2}\left(\theta-\theta_{0}\right)\right]\right\}
\end{aligned}
$$


Before we use (3.14) and (3.17) to calculate various propagators of interest we perform a second consistency check by noting that the sum $E+O$ should be equal to the probability of any Brownian motion path in this absorbing medium to survive at least till time $t$. According to (3.14) and (3.17) this probability is given by

$$
\begin{aligned}
E\left(r, \theta, t \mid r_{0},\right. & \left.\theta_{0}\right)+O\left(r, \theta, t \mid r_{0}, \theta_{0}\right) \\
= & z^{\prime \prime} \exp \left[z \cos \left(\theta-\theta_{0}\right)-z^{\prime}\right] \\
= & \frac{\omega}{\pi}\left(\frac{m}{8 D}\right)^{1 / 2} \operatorname{cosech}\left[(2 m D)^{1 / 2} \omega t\right] \\
& \times \exp \left[-\omega\left(\frac{m}{8 D}\right)^{1 / 2} \frac{\left(r_{0}^{2}+r^{2}\right) \cosh \left[(2 m D)^{1 / 2} \omega t\right]-2 r_{0} r \cos \left(\theta-\theta_{0}\right)}{\sinh \left[(2 m D)^{1 / 2} \omega t\right]}\right]
\end{aligned}
$$

The right-hand side indeed gives the proper expression for the propagator of a Brownian particle in a medium which can annihilate the particle with a probability $\frac{1}{2} m \omega^{2} r^{2}$ per unit of time.

\section{Calculation of the propagator}

Next we ask for the propagator of a Brownian particle which diffuses in the $x, y$ plane, in the case in which (i) there is a medium which can annihilate the particle with probability $\frac{1}{2} m \omega^{2} r^{2}$ per unit of time, and (ii) the branchline $T$ is an absorbing or a reflecting barrier for the particle. This propagator, which will be denoted by $u$, must be the solution of

$$
\partial u / \partial t=D \Delta u-\frac{1}{2} m \omega^{2} r^{2} u \quad(t>0)
$$

with the initial condition

$$
u(x, y, 0)=\delta\left(x-x_{0}\right) \delta\left(y-y_{0}\right)
$$

and with the Dirichlet boundary condition

$$
u(x, 0, t)=0 \quad(x<0, t>0)
$$

when the branchline is absorbing. For a reflecting branchline (4.3) should be replaced by the Neumann boundary condition

$$
\frac{\partial u}{\partial y}(x, 0, t)=0 \quad(x<0, t>0) .
$$

Using the analysis of the previous section one can immediately write down the explicit solution of this problem in terms of the total contributions of even and odd Brownian motion paths. Indeed, by changing to polar coordinates the solution of the problem with the Dirichlet boundary condition (4.3) is

$$
u(r, \theta, t)=E\left(r, \theta, t \mid r_{0}, \theta_{0}\right)-O\left(r, \theta, t \mid r_{0},-\theta_{0}\right)
$$

and the solution of the problem with the Neumann boundary condition (4.4) is

$$
u(r, \theta, t)=E\left(r, \theta, t \mid r_{0}, \theta_{0}\right)+O\left(r, \theta, t \mid r_{0},-\theta_{0}\right) .
$$

Note the appearance of $-\theta_{0}$ instead of $\theta_{0}$ in the second term on the right-hand side of (4.5) and (4.6). The correctness of (4.5) and (4.6) is obvious from the following three observations. 
(a) The functions $E\left(r, \theta, t \mid r_{0}, \theta_{0}\right)$ and $O\left(r, \theta, t \mid r_{0},-\theta_{0}\right)$ are sums of functions (3.5) which satisfy (3.3); hence, both $E$ and $O$ are solutions of the differential equation (4.1).

(b) In the limit $t \rightarrow 0$ the function $E$ tends to $\delta\left(x-x_{0}\right) \delta\left(y-y_{0}\right)$ and $O$ tends to 0 because of (3.4), hence (4.5) and (4.6) satisfy the initial condition (4.2).

(c) In the limit $\theta \rightarrow \pm \pi$ there exists a one-to-one correspondence between the even paths from $\left(r_{0}, \theta_{0}\right)$ to $(r, \theta)$ and the odd paths from $\left(r_{0},-\theta_{0}\right)$ to $(r, \theta)$ because these paths can be mapped onto each other by reflection with respect to the $x$ axis. So

$$
\begin{aligned}
& \lim _{\theta \rightarrow+\pi} E\left(r, \theta, t \mid r_{0}, \theta_{0}\right)=\lim _{\theta \rightarrow+\pi} O\left(r, \theta, t \mid r_{0},-\theta_{0}\right) \\
& \lim _{\theta \rightarrow-\pi} E\left(r, \theta, t \mid r_{0}, \theta_{0}\right)=\lim _{\theta \rightarrow-\pi} O\left(r, \theta, t \mid r_{0},-\theta_{0}\right) .
\end{aligned}
$$

Similarly

$$
\begin{aligned}
& \lim _{\theta \rightarrow+\pi} O\left(r, \theta, t \mid r_{0}, \theta_{0}\right)=\lim _{\theta \rightarrow+\pi} E\left(r, \theta, t \mid r_{0},-\theta_{0}\right) \\
& \lim _{\theta \rightarrow-\pi} O\left(r, \theta, t \mid r_{0}, \theta_{0}\right)=\lim _{\theta \rightarrow-\pi} E\left(r, \theta, t \mid r_{0},-\theta_{0}\right) .
\end{aligned}
$$

The last four equations imply that (4.5) obeys the absorbing boundary condition (4.3) and that (4.6) obeys the reflecting boundary condition (4.4).

By substitution of (3.14) and (3.15) into (4.5) and (4.6) one finds for the propagator the expression

$$
\begin{aligned}
& u(r, \theta, t)=\frac{1}{2} z^{\prime \prime} \exp \left[z \cos \left(\theta-\theta_{0}\right)-z^{\prime}\right]\left\{1+\operatorname{erf}\left[(2 z)^{1 / 2} \cos \frac{1}{2}\left(\theta-\theta_{0}\right)\right]\right\} \\
& \mp \frac{1}{2} z^{\prime \prime} \exp \left[z \cos \left(\theta+\theta_{0}\right)-z^{\prime}\right]\left\{1-\operatorname{erf}\left[(2 z)^{1 / 2} \cos \frac{1}{2}\left(\theta+\theta_{0}\right)\right]\right\}
\end{aligned}
$$

where the - sign is for the Dirichlet boundary condition and the + sign for the Neumann boundary condition.

Finally we observe that the transformations

$$
\begin{aligned}
& D \rightarrow \hbar^{2} / 2 m \\
& t \rightarrow \mathrm{i} t / \hbar \\
& u \rightarrow \psi
\end{aligned}
$$

will transform (4.1) into the Schrödinger equation

$$
i h \frac{\partial \psi}{\partial t}=-\frac{\hbar^{2}}{2 m} \Delta \psi+\frac{1}{2} m \omega^{2} r^{2} \psi
$$

The function $\psi$ obeys the correct initial condition and Dirichlet or Neumann boundary conditions on the branchline $T$. Hence $\psi$ is the propagator $g\left(r, \theta, t \mid r_{0}, \theta_{0}\right)$ of the quantum mechanical problem in (1.2). Explicit substitution gives

$$
\begin{aligned}
g\left(r, \theta, t \mid r_{0}, \theta_{0}\right) & \\
= & \frac{m \omega}{2 \pi \mathrm{i} h \sin \omega t} \exp \left(\frac{m \omega \mathrm{i}}{2 \hbar}\left(r_{0}^{2}+r^{2}\right) \cot \omega t\right) \\
& \times\left\{\exp \left(\frac{m \omega r_{0} r \cos \left(\theta-\theta_{0}\right)}{\mathrm{i} \hbar \sin \omega t}\right) F\left[\left(\frac{2 m \omega r_{0} r}{\hbar \sin \omega t}\right)^{1 / 2} \cos \frac{1}{2}\left(\theta-\theta_{0}\right)\right]\right. \\
& \left.\mp \exp \left(\frac{m \omega r_{0} r \cos \left(\theta+\theta_{0}\right)}{\mathrm{i} \hbar \sin \omega t}\right) F\left[-\left(\frac{2 m \omega_{0} r_{0} r}{\hbar \sin \omega t}\right)^{1 / 2} \cos \frac{1}{2}\left(\theta+\theta_{0}\right)\right]\right\}
\end{aligned}
$$


in terms of the Fresnel integral

$$
F(\xi)=\frac{1}{(\mathrm{i} \pi)^{1 / 2}} \int_{-\infty}^{\xi} \mathrm{e}^{\mathrm{i} \eta^{2}} \mathrm{~d} \eta .
$$

Here the - sign is for the Dirichlet boundary condition which is appropriate for a hard wall (the + sign is for the Neumann boundary condition, which seems not to occur in quantum mechanics).

\section{Concluding remarks}

The propagator (4.12), with the minus sign, describes the quantum mechanics of the simplest possible pinball machine in which a particle is caught in a harmonic field but is frustrated in its oscillations by the presence of a single impenetrable screen. According to classical mechanics the particle suffers collisions with the screen at times which are equally spaced with an interval $T=2 \pi / \omega$. The main feature added by quantum mechanics is the diffraction of the de Broglie waves around the edge of the screen, as represented by the two Fresnel integrals.

The exact result (4.12) seems to have a certain non-trivial richness of structure due to the two-valuedness of the square roots in the Fresnel integrals. We hope it will turn out to be of use as a laboratory in which various ideas can be tested, e.g. ideas related to the classical limit, asymptotic expansions, spreading of wave packets and various forms of perturbation theory.

The calculated propagator is an example of a quantum mechanical Green function which can be evaluated analytically, but for which the sum over classical paths does not lead to the exact result. Schulman (1982) has recently considered the SommerfeldCarslaw diffraction problem (our problem without the harmonic force) and showed that the exact propagator is found if one uses the sum over classical paths with one intermediate time. At the time of writing it is not clear if $(4.12)$ has the same property.

\section{References}

Abramowitz M and Stegun I A 1970 Handbook of Mathematical Functions (New York: Dover) van Andel P W 1986 unpublished Inomata A and Singh V A 1978 J. Math. Phys. 192318

Schulman L S 1982 Phys. Rev. Lett. 49599

Tanikella $V$ and Inomata A 1982 Phys. Lett. 87A 196

Wiegel F W 1977 J. Chem. Phys. 67469

1983 Phase Transitions and Critical Phenomena vol 7, ed C Domb and J L Lebowitz (New York: Academic) p 101

1986 Introduction to Path Integral Methods (Singapore: World Scientific)

Wiegel F W and Boersma J 1983 Physica 122A 325 